\title{
Modeling Buffer-Aided Relay Selection in Networks with Direct Transmission Capability
}

\author{
Themistoklis Charalambous, Member, IEEE, Nikolaos Nomikos, Student Member, IEEE, Ioannis Krikidis, \\ Senior Member, IEEE, Demosthenes Vouyioukas, Member, IEEE, and Mikael Johansson, Member, IEEE.
}

\begin{abstract}
We consider a wireless relay network that consists of a source, half-duplex decode-and-forward buffer-aided relays and a destination. While the majority of previous works on relay selection assume no direct transmission between source and destination in such a setting, we lift this assumption and propose a link selection policy that exploits both the buffering ability and the opportunity for successful reception of a packet directly from the source. The proposed relay selection scheme incorporates the instantaneous strength of the wireless links and adapts the relay selection decision based on the strongest available link. The evolution of the network as a whole is modeled by means of a Markov chain and thus, the outage probability is associated with the steady state of the Markov chain. It is deduced that even if the link between the source and the destination is in principle a very unreliable link, it is always beneficial for the source to multicast a packet to both the relay with the strongest available link and the destination.
\end{abstract}

Index Terms-Cooperative networks, relay channel, bufferaided relays, direct transmissions, outage probability.

\section{INTRODUCTION}

In classical relay selection policies, the source and the selected relay are assumed to be transmitting in orthogonal time-slots. Data buffers have been introduced at the relay nodes (see, e.g., [1]) allowing the selection of a different relay for reception and transmission in order to enhance the diversity gain. Despite the efficient use of the channel fading and the related performance benefits, relay selection policies are associated with a two-slot cooperative protocol where the schedule for the source and relay transmission is fixed a priori, and therefore, the available diversity degrees are not fully exploited. This limitation is relaxed in [2] where each slot is allocated dynamically to the source or a relay transmission, according to the instantaneous link quality and the status of the relays' buffers. More specifically, the proposed max - link relay selection scheme exploits fully the flexibility offered by the buffers at the relay nodes and at each time-slot selects the strongest link for transmission (source or relay transmission) among the available links. In [2], it is assumed that there is no direct link between the source and the relay.

In this work, we consider the relay selection problem in a network with multiple half-duplex buffer-aided relays and

T. Charalambous and M. Johansson are with the Automatic Control Lab, Electrical Engineering Department and ACCESS Linnaeus Center, Royal Institute of Technology (KTH), Stockholm, Sweden (E-mails: \{themisc, mikaelj\} akth.se).

N. Nomikos and D. Vouyioukas are with the Department of Information and Communication Systems Engineering, University of the Aegean,Karlovassi 83200, Samos, Greece (E-mails: \{nnomikos, dvouyiou \}aegean.gr).

I. Krikidis is with the Electrical and Computer Engineering Department, University of Cyprus, Nicosia 1678 (E-mail: krikidis@ucy.ac.cy). direct connectivity between the source and the destination. The contributions of the paper are the following:

(i) A new relay selection policy, herein called the modified max - link, is proposed; it allows the source to multicast the signal to a relay and the destination, a scenario which is more realistic than assuming there is no direct link.

(ii) The benefits of the direct link are clearly depicted via illustrative examples, in which the performance of our relay selection policy in terms of outage probability, average throughput and average delay is compared with max - link and other existing approaches.

(iii) The modeling and analysis of the direct transmissions from the source to the destination and transmission via multiple buffer-aided relays is theoretically investigated for the first time in this paper; for a single relay network, in terms of throughput maximization, the problem was investigated by e.g., [3], [4]. What facilitates this analysis is a model that combines the evolution of both the relay buffers and direct transmissions. We propose a way to construct the state transition matrix of the Markov chain (MC) of this combination and obtain the steady state with which we can characterize the outage probability. The new relay selection policy is analyzed in terms of outage probability and diversity. The benefits of the new protocol are corroborated in the numerical examples.

\section{SySTEM MODEL AND PRELIMINARIES}

We assume a simple cooperative network consisting of one source $S$, one destination $D$ and $K$ Decode-and-Forward (DF) relays $R_{k}(1 \leq k \leq K)$. All nodes are characterized by the half-duplex constraint and hence, they cannot transmit and receive simultaneously. A direct link between the source and the destination exists apart from the communication established via relays. Each relay $R_{k}$ holds a buffer (data queue) of capacity $L$ (number of data elements) where it can store source data that has been decoded at the relay and can be forwarded to the destination. The parameter $l_{k} \in \mathbb{Z}_{+}, l_{k} \in[0, L]$ denotes the number of data elements that are stored in the buffer of relay $R_{k}$; at the beginning, each relay buffer is empty (i.e., $l_{k}=0$, for all $k$ ). We denote by $\mathcal{T}$ all the relays for which their buffer is not empty and they can therefore transmit packets (i.e., $\mathcal{T}=\left\{R_{k}: l_{k}>0\right\}$, and by $\mathcal{A}$ all the relays for which their buffer is not full and hence they are available to receive a packet (i.e., $\mathcal{A}=\left\{R_{k}: l_{k}<L\right\}$ ). The buffer size $l_{k}$ is increased by one when a source packet (data) is correctly decoded at the $k$-th relay (and not received directly from the source), and it is decreased by one in case of a successful $k$-th relay transmission. 
Time is slotted and each transmitter (either the source $S$ or a relay $R_{k}$ ) transmits with a fixed power $P$. The source node is assumed to be saturated (infinite data backlog at the source). The retransmission process is based on an Acknowledgement/Negative-Acknowledgement (ACK/NACK) mechanism, in which short-length error-free packets are broadcasted by the receivers over a separate narrow-band channel.

Here, we assume that the clustered relay configuration ensures independent and identically distributed (i.i.d.) channel links [5]. This assumption simplifies the analysis and it is used in several studies in the literature [6]-[8]. In some occasions, it can be implemented by an appropriate long-term routing process which selects the clustered nodes to be close together [7], [8] or by power control [9] in order to improve diversity and hence, reduces outage events. If, however, none of the above approaches is facilitated and the links are non-i.i.d., then the buffers may become empty or full; this is a limitation of the proposed buffer-aided relaying protocol in this paper. All wireless links, including the $S \rightarrow D$ link, exhibit Rayleigh fading and are modeled as Additive White Gaussian Noise (AWGN). The fading coefficients $h_{i j}$ (for link $i \rightarrow j$ ) remain constant during a slot, but change independently from one slot to another according to a circularly symmetric complex Gaussian distribution with zero mean and unit variance. Furthermore, the variance of the AWGN is assumed to be normalized with zero mean and unit variance and therefore the SNR for each link is equal to $P$.

Each link $i \rightarrow j$ is characterized by the success probability $p \triangleq \mathbb{P}\left(P\left|h_{i j}\right|^{2} \geq \gamma_{0}\right)$ which denotes the probability that the link $i \rightarrow j$ is not in outage $(\bar{p}=1-p$ denotes the outage probability). An outage occurs when the instantaneous SNR of the link $i \rightarrow j$ is lower than a threshold $\gamma_{0}$. The implementation of the proposed scheme requires global CSI of the instantaneous channels and the buffer states. The central unit (e.g., destination) uses this information in order to select the appropriate relay node. This global CSI requires a continuous feedback for each wireless link as we use a continuous monitoring of the ACK/NACK signaling in order to identify the status of the buffers.

\section{The Link Selection Policy}

In [2] a relay selection policy is proposed where, at each time slot, it selects the strongest link for transmission (source or relay transmission) among the available links. Note that a source-relay link is considered to be available when the corresponding relay node does not have a full buffer and it can therefore receive data from the source, while a relaydestination link is considered to be available when the buffer of the relay node is not empty and it can transmit source's data towards the destination. If a source-relay link is the strongest link, the source transmits and the corresponding relay is selected for reception; on the other hand, if a relaydestination link is the strongest link, the corresponding relay is selected for transmission. However, it is assumed that there can never exist a source-destination $(S \rightarrow D)$ link.

The link selection policy that we propose a relay selection policy takes into account the possibility that some packets can be successfully delivered to the destination directly, i.e., if a source-relay link is the strongest link, the source multicasts the packet to the corresponding relay selected for reception and the destination. If the destination receives the packet successfully, an ACK is sent to the selected relay for reception during a dedicated ACK slot for the destination. In turn, the corresponding relay drops the packet and sends an ACK to the source (right after the ACK from the destination) that the packet was successfully received (no details needed to be specified). If the destination does not receive the packet successfully, a NACK is sent to the relay at the specific ACK slot. Hence, if the relay received the packet successfully, it sends an ACK (after the NACK) to the source and inserts the packet in the data queue. The $S \rightarrow D$ link does not take part in the selection process; hence, in each time slot, a link is chosen among the $2 K$ available links, i.e., the complexity of the relay selection procedure is $2 K$. It will be shown in the simulations that even if the link between the source and the destination is in principle a very unreliable link, it is always beneficial for the source to multicast a packet to both the relay with the strongest available link and the destination.

\section{OUTAGE PROBABILITY ANALYSIS}

The theoretical framework introduced in [2] is extended to account for direct transmissions from the source to the destination of the proposed link selection policy.

Definition of the outage probability. For the transmission from the source, the outage probability is defined as the probability that both the selected $S R$ and $S D$ links are in outage, i.e., $P_{\text {out }}^{S} \triangleq \mathbb{P}\left(P g_{S R^{*}}<\gamma_{0}\right) \mathbb{P}\left(P g_{S D}<\gamma_{0}\right)$, where $g_{i j} \triangleq\left|h_{i j}\right|^{2}$. For the transmission from the selected relay node, the outage probability is defined as the probability that the selected RD link is in outage, i.e., $P_{\text {out }}^{R} \triangleq \mathbb{P}\left(P g_{R^{*} D}<\gamma_{0}\right)$.

States of the MC. To represent the states of the MC we combine (i) the possible states of the buffers and (ii) the reception of a packet at the destination directly from the source. The transitions between the states are given by the probabilities of successful transmissions of packets. What is different to the analysis in [2] a state of the MC is represented not only by the number of elements at each buffer, but it also includes a state which depicts the reception of a packet directly from the source at the destination, denoted by $S_{D}$. The state of the MC can be represented by $S_{r+r_{\max } S_{D}} \triangleq\left(S_{D} l_{1} l_{2} \ldots l_{K}\right)$, where $r \in \mathbb{N}_{+}, 1 \leq r \leq r_{\max }, r_{\max } \triangleq(L+1)^{K}$, and $S_{D} \in\{0,1\}$ changes state every time a packet is received directly from the source, i.e., if $S_{D}$ is in state 1 and receives a packet directly from the source then it moves to state 0 , and vice versa. Note that state $S_{D}$ does not change if a packet is received by a relay, or, by the destination from a relay node.

Construction of the state transition matrix of the MC. Let $\mathbf{A} \in \mathbb{R}^{2 r_{\max } \times 2 r_{\max }}$ denote the state transition matrix of the $\mathrm{MC}$, in which the entry $\mathbf{A}_{i, j}=\mathbb{P}\left(S_{j} \rightarrow S_{i}\right)=$ $\mathbb{P}\left(X_{t+1}=S_{i} \mid X_{t}=S_{j}\right)$ is the transition probability to move from state $S_{j}$ at time $t$ to state $S_{i}$ at time $(t+1)$. In order to construct the state transition matrix $\mathbf{A}$, we need to identify the connectivity between the different states of the buffers. For each time slot, the buffer status can be modified as follows: (a) 
the number of elements of one relay buffer can be decreased by one, if a relay node is selected for transmission and the transmission is successful, (b) the number of elements of one buffer can be increased by one, if the source node is selected for transmission and the transmission to the relay is successful and the transmission to the destination is unsuccessful, (c) the buffer state (not the MC state) remains unchanged when either (i) there is a successful direct transmission or (ii) an outage event (i.e., either the source has been selected for transmission and the selected source-relay link and the source-destination link are in outage, or, a relay has been selected for transmission and the relay-destination link is in outage). As a result, the total number of available links, $D_{l}$, at each state $S_{j}$, is equal to $D_{l} \triangleq \sum_{i=1}^{K} \Phi\left(l_{i}\right)+1$, where

$$
\Phi\left(l_{i}\right)= \begin{cases}2, & \text { if } 0<l_{i}<L \\ 1, & \text { elsewhere }\end{cases}
$$

Note that in this case we have an extra link for each state $S_{l}$, compared to the max - link, due to the direct link between the source and the relay. In what follows, we distinguish the outage events at each communication link: the outage event $A$ denotes the case of experiencing an outage in either the $S R$ or the $R D$ links, while the outage event $B$ denotes the case of experiencing an outage in the $S D$ link. As a result, the probability of being in outage, while at stage $S_{l}$ is $\bar{p}_{l}=\mathbb{P}(A)^{D_{l}-1} \mathbb{P}(B)$. Note that the outage of the $S D$ link is different than that of the $S R$ and $R D$ links because the $S D$ link is not symmetric with respect to the other links. Even though the transition probabilities are affected, the structure of the Markov chain remains unchanged. Note that the MC used in [2] can also be used, but the probability of direct transmission has to be subtracted by the probability that the state of the MC will remain the same, when computing the outage probability.

Derivation of the outage probability. Due to the fact that the MC is Stationary, Irreducible and Aperiodic (SIA), a steady state $\boldsymbol{\pi}$ exists, i.e., $\mathbf{A} \boldsymbol{\pi}=\boldsymbol{\pi}$. The MC is constructed in a way that an outage event occurs only when there is no change in the buffer state. Hence, the outage probability of the system is given by the sum of the product of the probabilities of being at a stage $i$ and having an outage event, i.e.,

$$
P_{\text {out }}=\sum_{i=1}^{2 r_{\max }} \boldsymbol{\pi}_{i} \bar{p}_{i}=\operatorname{diag}(\mathbf{A}) \boldsymbol{\pi} .
$$

Eq. (2) shows that the construction of the state matrix $\mathbf{A}$ and the computation of the related steady state $\pi$ comprises a simple theoretical framework for the computation of the outage probability for the proposed relay selection policy. Due to the symmetry of the two-level MC, it can be deduced that $\pi$ is half the corresponding steady state of the MC in [2]; this can be used to reduce the computations, if it is an issue.

\section{ILLUSTRATIVE EXAMPLES}

A simple example with $K=2$ relays and $L=2$. We consider a simple clustered topology with $K=2$ relays and $L=2$. The state MC that captures both the evolution of the buffers and the packet reception at the destination directly from

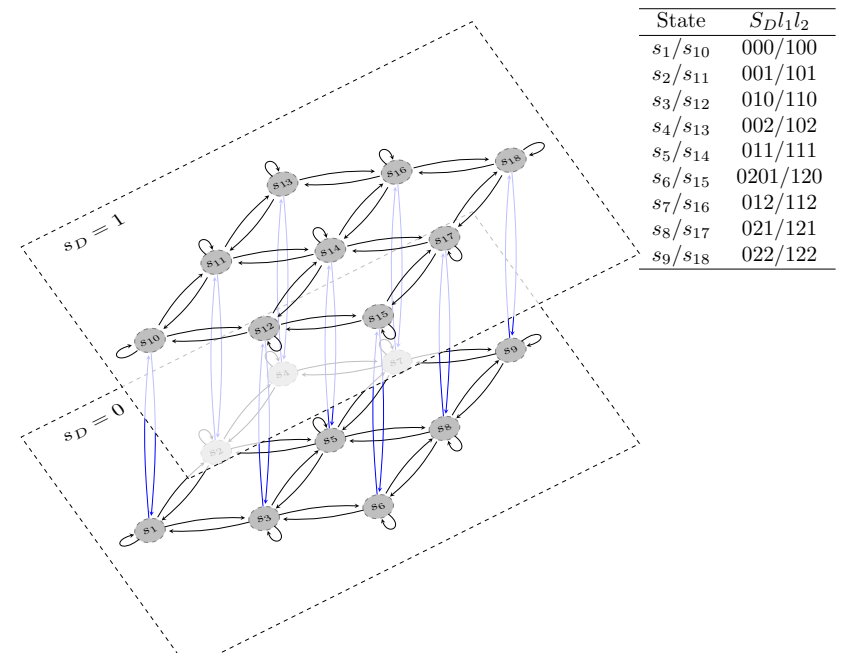

Fig. 1. State diagram of the MC representing the states of the network for $K=2$ relays and maximum queue size $L=2$ : the element $S_{D}$ shows the level of the MC and elements $l_{1}$ and $l_{2}$ show the buffer states of relays $R_{1}$ and $R_{2}$, respectively.

the source is presented in Fig. 1. In Fig. 1 the MC has two levels; the classical max - link corresponds to only one level of the MC. In each of these levels the transitions represent the evolution of the buffers. The state changes level when there is a direct transmission from the source to the destination; the new state corresponds to the equivalent state of the other level with the same state at the buffers. This approach allows for a simple derivation of the outage probability, since the system is in outage when the state in the MC remains unchanged.

Buffer size $L \rightarrow \infty$ and transmission power $P \rightarrow \infty$. As previously mentioned, the MC can also be represented as in [2]. Thus, by [2, Remark 1] it can be easily deduced that for $L \rightarrow \infty$ and $P \rightarrow \infty$ the dominant states are those for which the relays are neither full nor empty (i.e., $\left|\mathcal{T}_{i}\right|=\left|\mathcal{A}_{i}\right|=$ $K)$. Hence, the outage probability (i.e., the probability that all $2 K+1$ possible links are in outage) is given by

$$
\begin{aligned}
\bar{p}_{i j} & =p(A)^{2 K} p(B)=\left(1-\exp \left(-\frac{\gamma_{0}}{P}\right)\right)^{2 K}\left(1-\exp \left(-\frac{\gamma_{0}}{P}\right)\right) \\
& =\left(1-\exp \left(-\frac{\gamma_{0}}{P}\right)\right)^{2 K+1}
\end{aligned}
$$

The diversity order of the modified max - link, based on (3), is equal to $2 K+1$ :

$$
\begin{aligned}
d & =-\lim _{P \rightarrow \infty} \frac{\log \mathbb{P}_{\text {out }}(P)}{\log P}=-\lim _{P \rightarrow \infty} \frac{\log \left(1-\exp \left(-\frac{\gamma_{0}}{P}\right)\right)^{2 K+1}}{\log P} \\
& \stackrel{(a)}{\approx}-(2 K+1) \lim _{P \rightarrow \infty} \frac{\log \left(\frac{\gamma_{0}}{P}\right)}{\log P}=2 K+1
\end{aligned}
$$

Approximation $(a)$ emerges from: $x \rightarrow 0$, then $1-e^{-x} \approx x$.

\section{NUMERICAL RESULTS}

The performance of the modified max - link, is evaluated here, in terms of: (a) outage probability, (b) average throughput and (c) average delay, between the Hybrid Relay Selection (HRS) proposed in [1], and the modified max - link, for varying $S \rightarrow D$ channel power, as well as without the $S D$ link (classical $\max -\operatorname{link}$, i.e., $\left|h_{S D}\right|^{2}=0$ ). For the results, we 
have assumed a per-link outage threshold of 1 bit per channel use (BPCU) corresponding to an SNR equal to $\gamma_{0}=0 \mathrm{~dB}$.

Outage Probability. In Fig. 2, it is observed that HRS has the worst performance as only two links are available when the buffer is neither full nor empty. For the modified max - link curves, as the $S \rightarrow D$ link's condition improves, so do the diversity and coding gains as an extra link is available for transmission with higher probability. Also, we provide the curve of the selection bound (assumes that the relays' buffers are never empty of full) given by simulation and theoretical analysis. For high SNR there is extra diversity gain and the better the $S \rightarrow D$ link the higher the coding gain.

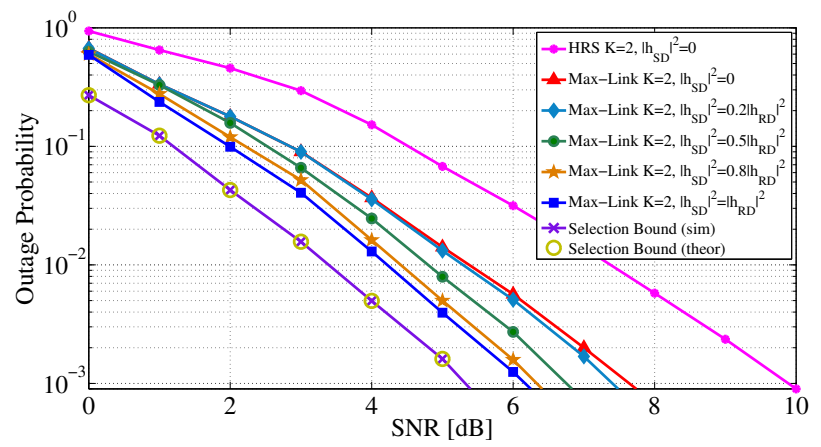

Fig. 2. Outage Probability for increasing transmit SNR. In this example, $K=2$ and $L=2$.

Average Throughput. In Fig. 3 it is observed that HRS and the classical max - link (i.e., $\left|h_{S D}\right|^{2}=0$ ) can achieve a maximum throughput of 0.5 BPCU. On the contrary, the availability of the $S D$ link allows the full throughput to be achieved and as the maximum $S D$ channel power increases, the maximum throughput of 1 BPCU is reached faster. While the outage probability is slightly improved with the addition of the direct link, the throughput is improved considerably. Furthermore, one may see that the addition of extra relays provides improved throughput in the low SNR regime.

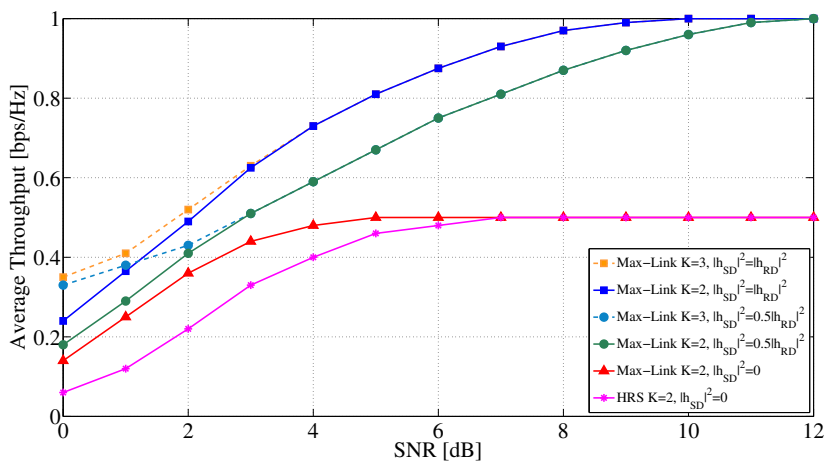

Fig. 3. Average throughput for $K=2,3$ and $L=2$.

Average Delay. The average delay is computed by considering the time a packet needs to reach the destination once it has left the source; consequently, direct transmission is assumed to have no delays and for packets that go through the relays, the delay of the packet is essentially the number of time slots the packet stays in the relay. The average delay is the total number of time slots required by all packets to reach the destination over the total number of time slots. The low diversity of HRS in the low SNR regime causes many instances where packets are trapped in the buffers. However, the max - link schemes

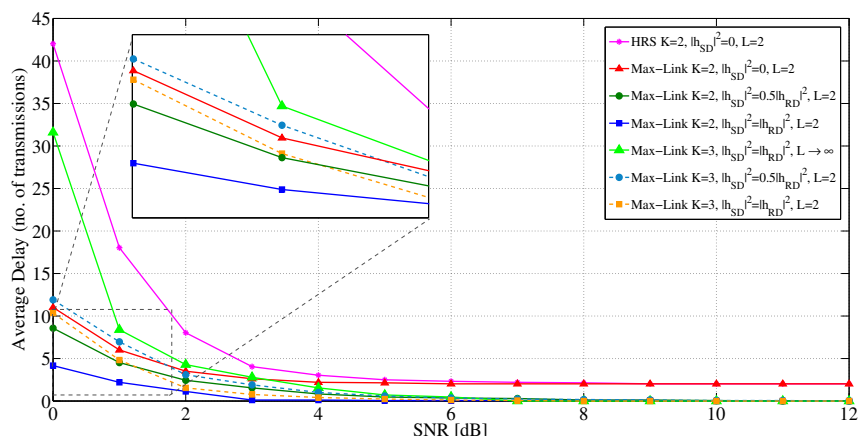

Fig. 4. Average delay for increasing transmit SNR, $K=2,3$ and $L=2, \infty$.

leverage this constraint through adaptive link selection. For high SNR values, the $S \rightarrow D$ transmissions become successful; thus, the average delay becomes 0. Employing more relays $(K=3)$ results in increased average delay in the low SNR regime as the probability to select a specific relay to transmit on the $R \rightarrow D$ link decreases. Finally, when $L \rightarrow \infty$, for good $S \rightarrow D$ link and high SNR values (above $4 \mathrm{~dB}$ ) the direct transmissions are dominant and the average delay diminishes.

\section{CONCLUSIONS}

We proposed a modified version of the max - link policy to account for the direct transmission from the source to the destination. The scheme incorporates the instantaneous strength of the wireless links and adapts the relay selection decision based on the strongest available link. The combination of direct transmissions from the source to the destination and transmission via buffer-aided relays shows that even if the link between the source and the destination is unreliable, it is always beneficial to aim to multicast a packet to both the relay with the strongest available link and the destination.

\section{REFERENCES}

[1] A. Ikhlef, D. S. Michalopoulos, and R. Schober, "Max-max relay selection for relays with buffers," IEEE Trans. Wireless Commun., vol. 11, pp. 1124-1135, March 2012.

[2] I. Krikidis, T. Charalambous, and J. S. Thompson, "Buffer-aided relay selection for cooperative diversity systems without delay constraints," IEEE Trans. Wireless Commun., vol. 11, pp. 1957-1967, May 2012.

[3] N. Zlatanov, R. Schober, and L. Lampe, "Buffer-Aided Relaying in a Three Node Network," IEEE Int. Symp. Inf. Theory, pp. 781-785, July 2012.

[4] A. El Shafie, M. Khafagy, and A. Sultan, "Optimization of a RelayAssisted Link with Buffer State Information at the Source," IEEE Commun. Letters, 2014 (to appear).

[5] K. -S. Hwang, Y. -C. Ko, and M. -S. Alouini, "Performance analysis of two-way Amplify-and-Forward relaying with adaptive modulation over multiple relay network," IEEE Trans. Wireless Commun., vol. 59, pp. 402-406, Feb. 2011.

[6] S. Yang and J. -C. Belfiore, "Towards the optimal amplify-and-forward cooperative diversity scheme," IEEE Trans. Inf. Theory, vol. 53, pp. 3114-3126, Sept. 2007.

[7] I. Krikidis, J. S. Thompson, S. McLaughlin, and N. Goertz, "Amplifyand-forward with partial relay selection," IEEE Commun. Lett., vol. 12, pp. 235-237, April 2008.

[8] D. Benevides da Costa and S. Aissa, "End-to-end performance of dualhop semi-blind relaying systems with partial relay selection," IEEE Trans. Wireless Commun., vol. 8, pp. 4306-4315, Aug. 2009.

[9] Z. Tian, G. Chen, Y. Gong, Z. Chen, J. Chambers, "Buffer-Aided MaxLink Relay Selection in Amplify-and-Forward Cooperative Networks," IEEE Trans. Vehicular Tech., 2014 (to appear). 